\title{
A COMPARATIVE STUDY OF VARIOUS WAVELET APPROACHES USED IN IMAGE DENOISING
}

\author{
Vijayaragahvan Veeramani1 ${ }^{*}$ and Laavanya Mohan ${ }^{1}$ \\ Vignan's Foundation for Science Technology and Research, \\ Vadlamudi, Guntur, Andhra Pradesh-522213, India. \\ vijayaragahvan123@gmail.com
}

\section{ABSTRACT:}

The world is constantly changing, and vision helps the humans to understand the environmental changes over time. The changes can be seen by, capturing the images. Hence digital image plays a vital role in day to day life. During the process of acquisition of digital image, the qualities of digital pictures are degraded due to additive noise known as adaptive white Gaussian noise. Therefore, the major challenge of image denoising algorithm is to improve the visual appearance while preserving the other details of the image. For the last two decades, wavelet has become an elegant tool in image denoising techniques. Among all wavelet based denoising methods, wavelet thresholding became popular because, wavelet appropriately separates the noisy signal from the image. The wavelet separation leaves the coarse grain noise in approximation sub-band and fine grain noise in detail sub-bands. Therefore, in wavelet based thresholding methods noise in detail sub-bands are threshold and approximate sub-band noise are kept as such. Hence, the efficiency of all wavelet based shrinkage techniques depends on, the choice of threshold parameter, thresholding technique and how the noise in the approximation subbands are handled. This paper presents a brief comparative study of denoising techniques proposed in the research articles based on the above parameters for Gaussian noise reduction using various wavelets transform. With the help of these experiments, we are able to identify the strengths and weaknesses of these methods, as well as seek the way ahead towards a definitive solution to the long-standing problem of image denoising.

KEYWORDS: Image denoising, Image processing, Thresholding function, Wavelet transform.

\section{INTRODUCTION}

The aim of digital image processing is to improve the potential information for human interpretation, processing image for storage and transmission. Among the different types of noises, Gaussian noise is considered by many researchers because; Gaussian noise arises during acquisition due to sensor noise caused by poor illumination. Gaussian noise disturbs the grey values in digital images. Generally Gaussian noise is considered as white Gaussian noise since, in white noise pixels are uncorrelated and the pixel values are identically distributed. The general block diagram of image denoising is shown in figure 1.

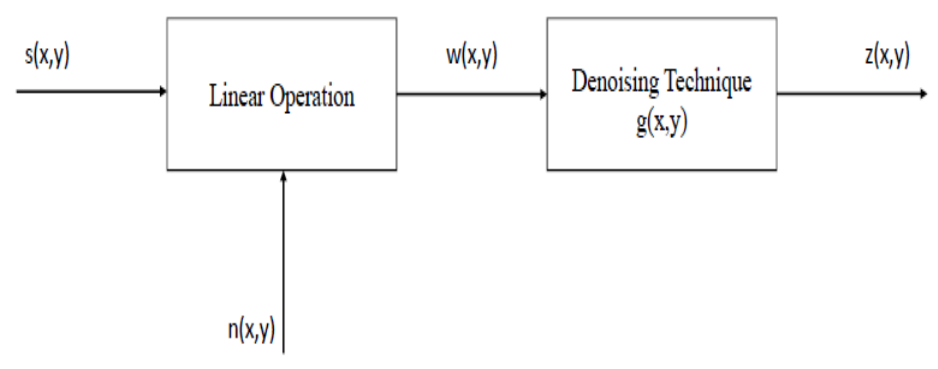

Fig. 0: Image Denoising 
In figure $1, s(x, y)$ is the original image, $n(x, y)$ is the noise that is added (or) multiplied in the linear operation block to the original image. This produces a noisy image $w(x, y)$. Now the next step is to apply a suitable denoising technique $g(x, y)$ to obtain a noise free image $z(x, y)$.

Noise is the most common problem in image processing. Image denoising is used to produce good estimates of the original image from noisy observations. Therefore a variety of methods have been proposed for image denoising. This includes spatial domain, transform domain and hybrid domain based methods.

Spatial domain methods use the spatial correlation of pixels to suppress noise. Spatial domain filters are further divided into local and non-local filters. The local bilateral filter proposed by Smith \& Brady [1] and Tomasi \& Manduchi [2] considers both spatial and intensity information to find the new pixel. The extension of bilateral filter was introduced by Buades et al. [3], is Non Local Mean filter (NLM) obtained by replacing the point wise photometric distances with patch distances. The NLM filter estimates each pixel value based on the Euclidean distance between the patch centered on the pixel being denoised and the neighbouring pixel. Zhang et al. [4] proposed a two-direction non-local variational model to improve the denoising performance, but it was time consuming. The fast weighted average computation using an integral image for NLM filter proposed by Cheng et al. [5] gives better denoising accuracy.

Transform domain methods use sparsity representation of image coefficients to distinguish the signal and noise. Among transform based methods wavelets introduced by Mallat [6] have been recognized as a powerful tool for analysis of signals and images. The wavelet transform based denoising schemes are broadly classified in to threshold based schemes and model based schemes. Wavelet transform are further classified into UDWT and DTCWT. UDWT in image denoising is computationally demanding than decimated transform. In undecimated transform the white noise in the input image remains white after the transform. The undecimated transform is shift invariant. DTCWT has both shift invariance and directional selectivity property. DTCWT has limited redundancy of $2^{\mathrm{d}}$ for $\mathrm{d}$ dimension. The combination of both UDWT and DTCWT gives exact shift variance and directional selectivity, was proposed by Hill et al. [7]. In UDTCWT phase offset is required to create the filter bank structure. This requirement leads to improper alignment of basis function of each individual component function with its parent.

The hybrid of spatial and transform domain based schemes have achieved great success in image denoising, because it uses both the spatial correlation and sparsity of image. The most well-known adaptive denoising scheme is the PCA with local pixel grouping introduced by Zhang et al. [8]. This approach uses the block matching to group pixels with similar structures and transform them using PCA basis and shrinks coefficients using the linear MMSE estimator. PCA is also used for hyper-spectral images along with bivariate shrinkage function gives satisfactory denoising results with small amount of artifacts in the denoised image was proposed by Chen \& Qian [9]. NLM filter in wavelet domain was projected by You \& Cho [10], uses noise statistics to change the kernel bandwidth for every sub-band. When NLM filter is applied on the approximation subband to remove the noise, the NLM decay parameter can be analyzed to enhance the 
performance was presented by Balasubramanian et al. [11]. Hence, the effective performance of the denoising technique depends, sparse representation of the image and correlation between the wavelet coefficients.

\section{RELATED WORKS}

In local denoising methods images are assumed to be true and noises to be spread in all frequencies. Gaussian noise can be removed, by varying the size of a window, based on region homogeneity was developed by Eom \& Kim [12]. A non-local method proposed by Buades et al. [13, 3] is based on the self-similarity between image pixels and it is called as NLM. The noise free pixel intensity is computed as a weighted average of all pixel intensities in the given image, and the weights are proportional to the similarity between the native neighbourhood of the pixel and its surrounding pixels. The NLM filter works by comparing the grey levels at a single point along with their geometrical configuration in the whole neighbourhood. Salmon [14] proposed a denoising method using NLM, here the weight of the central patch was identified with the help of SURE and suits well for moderate noise level. Zhang et al. [4] projected a noise reduction method with the help of, similarities between the patches along rows and columns. Xu et al. [15] proposed patch based non-local self with previous learning phase and the denoising phase. NLM filter blurs the image details when noise level is high. Tang \& Yang [16] introduced K-SVD combined with NLM filter for denoising image gives $2 \%$ extra performance than NLM filter. Cheng et al. [5] Proposed image denoising based on integral NLM provides denoising performance same as classical NLM but with computation time reduction. Image denoising using NLM and Improved Perona-Malik (IPM) denoise images by considering intensity values along with self-similarities of gradient and space position was proposed by Yuan [17].

Wavelet transform have properties like scarcity, energy compaction and multiresolution structure. Due to these properties wavelet has obtained ample interest in noise elimination was addressed by Elad \& Bruckstein [18]. Using wavelet, noise is taken away by killing the noisy coefficients with the help of threshold value, was proposed by Donoho \& Johnstone [19]. Later in wavelet threshold methods, countless works were carried out, to find the threshold value was suggested by [20, 21, and 22]. The name of the shrinkage functions are, VisuShrink which is based on universal threshold, SureShrink is built on Stein's Unbiased Risk Estimator (SURE) was given by Donoho \& Johnstone [19] and BayesShrink is a data-driven adaptive image denoising method given by Chang et al. [23]. In this method the image will be divided into sub-bands namely LL, LH, HL and HH. The approximation sub-band is kept as such and detail sub-bands are processed. Hence in this approach the coarse noise is maintained in approximation sub-band. The NLM algorithm when used in wavelet domain a relative improvement, in shrinking of wavelet coefficients is obtained was stated by You \& Cho [10]. But, all these wavelet methods suffer from shift invariance and directional selectivity. These limitations can be overcome by DTCWT introduced by Kingsbury [24].

All the above wavelet threshold based techniques eliminate noise without considering the inter-scale and intra-scale dependencies among wavelet coefficients. Mihcak et al. [25] projected each wavelet coefficients by a doubly stochastic process on local variance with the help of detected noisy data in a local neighbourhood. Formerly a 
rough mean square error estimation process was used to re-establish the noisy coefficients. Sendur \& Selesnick [26, 27] proposed a denoising way to estimate the wavelet coefficients depending on the parent coefficients. Shrinkage is better if the parent coefficient is smaller. Image denoising using local mixture in sparse domain provides good PSNR value and better visual appearance was addressed by Rabbani \& Gazor [28]. Hybrid neighbourhood filter can be used to denoise an image with high PSNR and less computational time was presented by Hussain \& Gorashi [29]).

In transform domain, using wavelet tool to denoise the image by modifying the wavelet coefficients gives numerous artifacts in image, due to shift variance and poor directional selectivity was addressed by Unser [30] and Rockinger [31]. In order to overcome this problem, Undecimated Discrete Wavelet Transform (UDWT) proposed by Gyaourova [32] use only upsampling and eliminates down sampling of the image, produces more precise information to denoise the image. The UDWT in remote sensing image denoising with the help of Bayesian shrinkage obtains good visual quality than DWT was introduced by Wang \& Li [33]. The problem with UDWT is the requirement of more storage space, computation. The UDWT also agonizes with over completeness and directional selectivity. This disadvantage can be overcome by the use of DTCWT with the properties of shift invariance and directional selectivity. Chen \& Qian [9] proposed a method to denoise a hyper-spectral image along with bivariate shrinkage function and PCA to improvise the noise reduction.

Achim \& Kuruoglu [34] developed a denoising method using bivariate maximum a posteriori estimator. This method trusts on isotropic $\alpha$-distribution, suffers with translation invariant in complex wavelet domain. Liu \& Jiang [35] proposed a DTCWT based denoising algorithm, where the variance is valued by seeing directional windows on local neighbourhood. The outcomes specify that, this technique is advanced by $0.7 \mathrm{~dB}$ PSNR than other existing methods. The disadvantage of this method is, when the real and imaginary coefficients relationships are altered, and then phase noise will be introduced. Chen et al. [36] introduced denoising technique with three scale in dual tree complex wavelet domain attains improved denoising performance. Varsha \& Preetha [37] presented a denoising method using DTCWT, it uses generalized cross validation to threshold the image provides a better PSNR value.

Modified threshold using wiener filter is used to remove the noise in the DTCWT sub band coefficients and it performs better than local wiener filter is addressed by Zhang [38]. Yaseena et al. [39] presented the purpose of this study is to compare image collision techniques based on real and complex wavelet transitions. Classical Discrete Waveform Transformations (DWT) has the potential to provide rigid and smooth limits. Image quality Standard 2-D DWT and Double-Tree Complex Wavelet Transform (DT-CWT) are studied and it shows that DTCWT improves 2-D DWT in the right choice of threshold. A new method of image denoise based on the use of median filter (MF) is presented by Ramadhan et al. [40]. Wavelet transforms on work frequencies of sub-bands divided by image are a powerful method for analyzing images. Accordingly experimental work shows that the proposed method yields better results than using only median filter only. The method proposed by Gajbhar and Joshi [41] involves the use of triple half-band Optimization of free variables obtained using Filter Bank and 
Factorization to create a two-tree filter of the generalized half-band polynomial DTCWT. The wave functions associated with these trees show good analysis in terms of qualitative and quantitative measures.

Hill et al. [7] presented a new transform named as UDTCWT, in this transform the resolution of the sub-band image is same the image with shift invariance. This transform also has a one-to-one relationship between co-located coefficients in all sub-bands and the image pixels. By using UDTCWT along with bivariate shrinkage using Cauchy model provides improved denoising enactment. The problem with UDTCWT is the phase offset requirement during the creation of filter bank structure. Hence an improper alignment of parent with its wavelet coefficients exists.

From the detailed study from the existing methods, that need attention for improving the denoising performance is divided in to three factors. First one is the selection of threshold value, since threshold plays the major role to make the denoised signal to fit the input level. Secondly, self-similarity between image pixels and inter-scale dependencies between wavelet coefficients have to be considered while threshold estimation. Third one is the lack of spatial and sub-band adaptivity of the denoising techniques. All the above mentioned factors are addressed by Vijayaraghavan \& Laavanya [42-45] for image denoising using various wavelet transforms is discussed in the below section.

\section{WAVELET TRANSFORM AND ITS APPLICATION TO IMAGE DENOISING}

\subsection{Locally adaptive window maximum likelihood and non-local means filter}

In this section a statistical model based denoising scheme is presented in wavelet domain. Here, the inter-scale dependency of the transform coefficient is considered in the modelling process, hence this method has sub-band adaptivity. Further the selfsimilarity between image pixel are also considered to enhance the denoising performance.

In this approach the denoised wavelet coefficients are obtained by using a statistical model, for estimating the threshold value. Under this framework, noise variance is computed by robust median estimator and signal variance is estimated using a Maximum Likelihood (ML) estimator through all scales of sub-band. Using the information of noise variance and signal variance, each wavelet coefficient is shrinkage with different threshold value. Therefore, the presented method has subband adaptivity. Non Local Mean (NLM) filter is employed to further improve the denoising performance.

Here, inter-scale dependency of transform coefficient using ML estimator incorporated with non-local mean filter is presented. This scheme is simple and computationally efficient with better performance.

\section{2. Real oriented 2-D DTWT with non- local means filter}

Here scale dependent soft thresholding multiresolution technique with noise variance estimation for denoising images is presented. In this method, self-similarity between wavelet coefficients is considered to denoise effectively by preserving the image details than DWT.

The RDTWT based denoising method presented here, is an extension of wavelet based denoising techniques. The RDTWT has six orientations and can be implemented using two real separable 2D wavelet transforms in parallel. RDTWT is used to represent the 
image sparsely with directional selectivity. In order to, average out the noise effectively NLM filter is used. NLM calculates the pixel weights of the image by considering all possible self-similarities of the image. Since NLM cares only self-similarity measure the key to the unification of universal measure is to use soft thresholding rule. Finally denoised image is reconstructed from the modified wavelet coefficients.

\section{3. DTCWT and non-local mean filter with Visushrink}

The major problem with threshold based methods is the choice of suitable threshold value. If threshold value is small, then noisy coefficients are surpassed. Conversely large threshold value makes more number of the wavelet coefficients as zero. In previous work, RDTWT has been used which has approximate shift invariance. The RDTWT imaginary coefficients are not considered. But, in DTCWT both real and imaginary coefficients are considered. Therefore, the combination of Dual Tree Complex Wavelet Transforms (DTCWT) and Non-Local Mean (NLM) filter along with VisuShrink for image denoising is presented.

Noise is the integral part of image processing applications. Hence noise has become the paramount concern. The presented method is very simple and elegant one by using DTCWT since, it has the properties of shift invariance and directional selectivity with limited redundancy. Here self-similarity between the sub-band coefficients are taken into account to reduce the low frequency noise. This reduction in noise is done by using NLM filter. To obtain a smooth denoised image with good visual appearance, each wavelet coefficient is thresholded using VisuShrink function.

\section{4. Undecimated DTCWT and principal component analysis}

An efficient sub-band adaptive denoising scheme using Undecimated Dual Tree Complex Wavelet Transform (UDTCWT) is presented. In this approach the parent (LL sub-band) and its wavelet coefficients are aligned properly using PCA. PCA projects the variance of wavelet coefficients in specific direction. The noise variance is estimated using robust median estimator. The presented scheme is sub-band adaptive because the signal variance is estimated by using a ML estimator through all scales of sub-bands and for each wavelet coefficient the signal variance is varied. Finally, each denoised wavelet coefficient is estimated using minimum mean square error function.

The effectiveness of the denoising performance depends on three factors. One is the efficient representation of noisy image with improved directionality and shift invariance, second one is to get compaction of signal energy in few principal components and the third one is the efficient computation of the signal and noise variance. In this work the first requirement is met using UDTCWT, the second one by using principal component analysis and the third one is by using locally adaptive window maximum likelihood estimator on the magnitude of wavelet coefficients.

\section{COMPARISION OF FOUR DENOISING APPROACHES IN WAVELET DOMAIN}

The performance of the presented methods is compared based on PSNR value, MSSIM, SSIM index map and by visual quality. The Lena, Boat, Barbara are the test images taken for simulation with size $512 \times 512$.

The comparison of PSNR value is shown in table 1. From the table 1, it can observe that the presented scheme-1 which is based on wavelet domain using NLM filter as post 
processing filter provides better denoising performance only when noise level is low. The scheme- 2 is in real dual tree wavelet domain performs better than discrete wavelet domain in quantitative analysis but it fails in visual quality. The third scheme is in DTCWT domain using VisuShrink, it gives competitive performance than discrete wavelet transform and real oriented dual tree due to the properties of approximate shift invariance and directional selectivity. The denoising ability can improvised if the transform has the advantage of both DTCWT and UDWT. One such transform is UDTCWT; this transform is used in the scheme-4 along with PCA using LAWML shrinkage function to give better denoising performance. The graphical comparison of PSNR values of Lena, Boat and Barbara are shown in figures 2, 3 and 4.

In general image quality measurement is related to image similarity measurement. Therefore, image quality of the presented methods is compared using mean Structural Similarity Index (SSIM). SSIM depends on the structural information from the view point. Hence, if change in structural information is found between distorted and original image that could be a distortion of the perceived image. SSIM is a full reference parameter. SSIM is a good metric than PSNR due to inconsistent with human visual perception. SSIM measures the structural information along with perceptual information's like luminance and contrast masking. Further the MSSIM gives better consistency with qualitative visual appearance.

MSSIM measure for all the presented methods are shown in table 2 . It is clear from the table 2 that among the presented methods UDTCWT along with PCA and LAWML shrinkage functions gives better visual quality than other methods. This is because,
UDTCWT has exact shift variance, good directional selectivity and along with PCA has the ability to maximize the wavelet energy of the sub-band. The graphical comparison of MSSIM measure for Lena, Boat and Barbara are shown in figures 5, 6 and 7.

To emphasize the visual quality of the denoised images of all four methods, images like Boat, Barbara and Lena for noise level 25 are shown in figures 8,9 and 10. All comparison ensures that denoising using UDTCWT-PCA is better in high noise environment than other denoising techniques.

Table 1: Comparison of PSNR values of the four denoising methods

\begin{tabular}{|c|c|c|c|c|}
\hline $\begin{array}{c}\text { Denoisin } \\
\text { g } \\
\text { Methods }\end{array}$ & $\begin{array}{l}\boldsymbol{\sigma} \\
\mathbf{n}\end{array}$ & $\begin{array}{c}\text { LEN } \\
\mathbf{A}\end{array}$ & $\begin{array}{c}\text { BOA } \\
\mathbf{T}\end{array}$ & $\begin{array}{c}\text { BARBA } \\
\text { RA }\end{array}$ \\
\hline \multirow{5}{*}{$\begin{array}{c}\text { SCHEME } \\
-1\end{array}$} & $\begin{array}{l}1 \\
0\end{array}$ & 34.80 & 32.40 & 32.74 \\
\hline & $\begin{array}{l}1 \\
5\end{array}$ & 33.05 & 30.71 & 30.53 \\
\hline & $\begin{array}{l}2 \\
0\end{array}$ & 31.75 & 29.47 & 29.00 \\
\hline & $\begin{array}{l}2 \\
5\end{array}$ & 30.67 & 28.44 & 27.75 \\
\hline & $\begin{array}{l}3 \\
0\end{array}$ & 29.81 & 27.59 & 26.92 \\
\hline \multirow{5}{*}{$\begin{array}{c}\text { SCHEME } \\
{ }_{2}^{2}\end{array}$} & $\begin{array}{l}1 \\
0\end{array}$ & 33.46 & 31.72 & 31.36 \\
\hline & $\begin{array}{l}1 \\
5\end{array}$ & 31.38 & 29.65 & 29.05 \\
\hline & $\begin{array}{l}2 \\
0\end{array}$ & 29.82 & 28.22 & 27.44 \\
\hline & $\begin{array}{l}2 \\
5\end{array}$ & 28.01 & 27.09 & 26.25 \\
\hline & $\begin{array}{l}3 \\
0\end{array}$ & 28.71 & 26.12 & 25.30 \\
\hline \multirow{2}{*}{$\begin{array}{c}\text { SCHEME } \\
{ }_{3}^{3}\end{array}$} & $\begin{array}{l}1 \\
0 \\
\end{array}$ & 33.80 & 30.74 & 30.59 \\
\hline & $\begin{array}{l}1 \\
5\end{array}$ & 32.23 & 29.15 & 28.38 \\
\hline
\end{tabular}




\begin{tabular}{|c|c|c|c|c|}
\hline & 2 & 30.97 & 28.00 & 26.93 \\
\cline { 2 - 5 } & 0 \\
2 & 3 & 30.03 & 27.16 & 25.84 \\
\cline { 2 - 5 } & $\begin{array}{c}3 \\
0\end{array}$ & 29.34 & 26.47 & 24.91 \\
\hline $\begin{array}{c}\text { SCHEME } \\
4\end{array}$ & 1 & 35.05 & 32.91 & 33.31 \\
\hline
\end{tabular}

\begin{tabular}{|l|l|l|l|l|}
\hline & 1 & 33.21 & 30.75 & 31.08 \\
\hline 5 & 2 & 31.80 & 29.54 & 29.57 \\
\cline { 2 - 5 } & & & \\
\cline { 2 - 5 } 2 & 30.75 & 28.49 & 28.58 \\
\hline 5 & & 27.75 & 27.55 \\
\hline $\begin{array}{l}3 \\
0\end{array}$ & 29.77 & 27 & \\
\hline
\end{tabular}

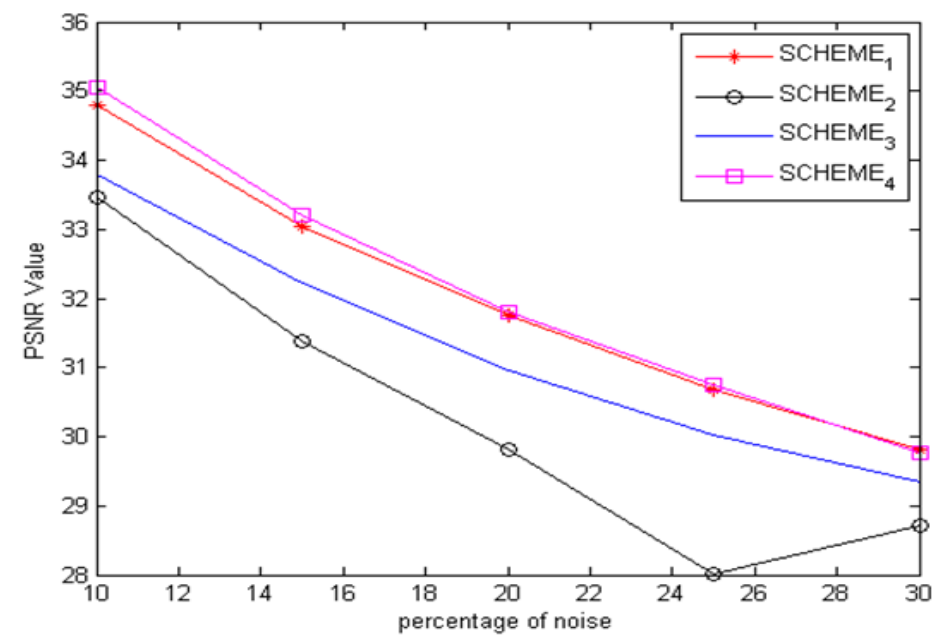

Fig. 2: PSNR values of four denoising methods of Lena image

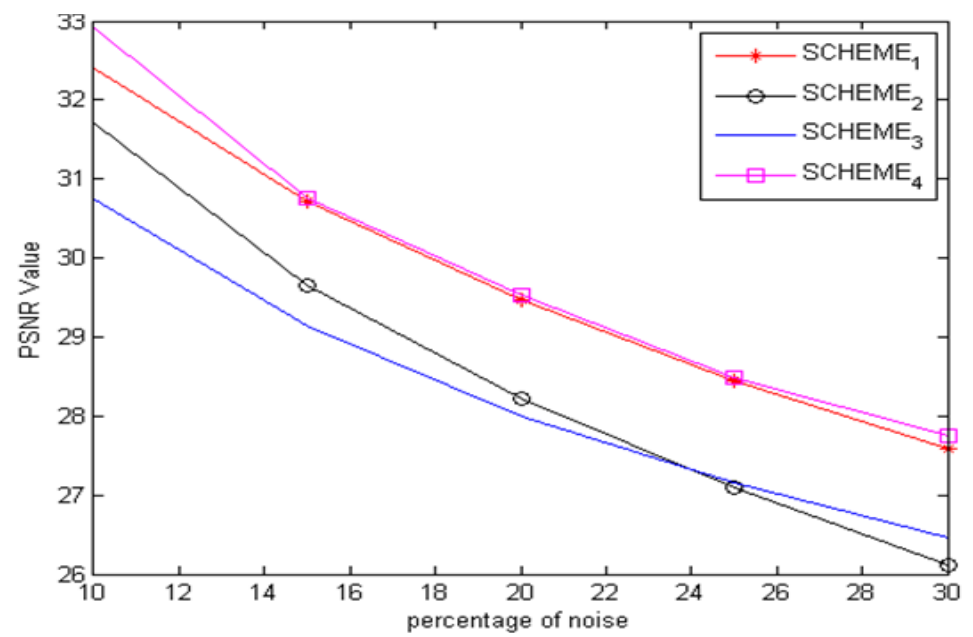

Fig. 3: PSNR values of four denoising methods of Boat image 


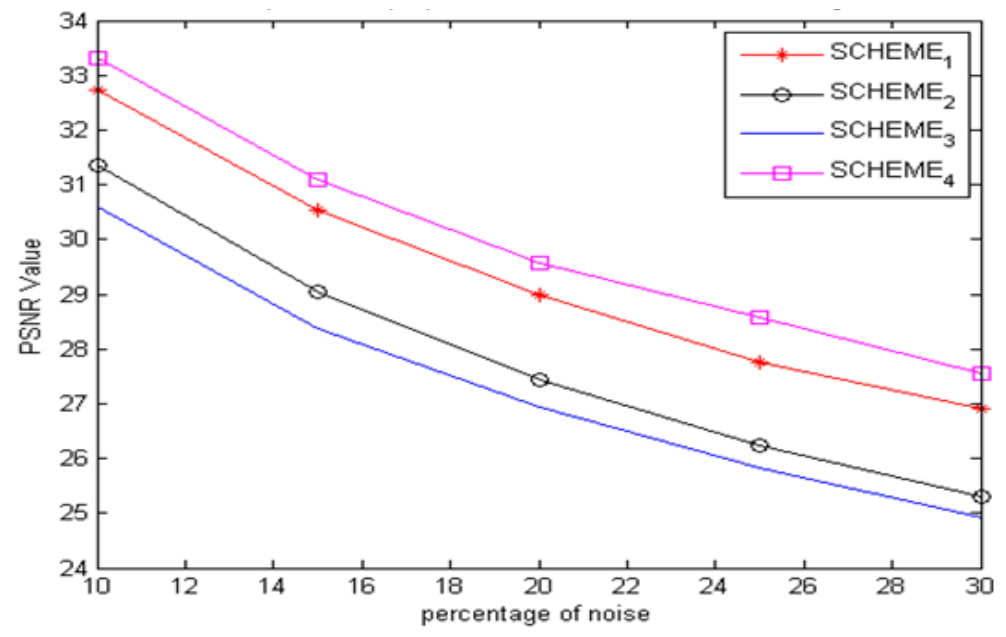

Fig. 4: PSNR values of four denoising methods of Barbara image

Table 2: Comparison of MSSIM values of the four denoising methods

\begin{tabular}{|c|c|c|c|c|}
\hline $\begin{array}{c}\text { Denoising } \\
\text { Methods }\end{array}$ & $\boldsymbol{\sigma}_{\mathbf{n}}$ & LENA & BOAT & BARBARA \\
\hline \multirow{4}{*}{ SCHEME_1 } & 10 & 0.8757 & 0.8469 & 0.9144 \\
\cline { 2 - 5 } & 15 & 0.8751 & 0.8149 & 0.8845 \\
\cline { 2 - 5 } & 20 & 0.8467 & 0.7859 & 0.8531 \\
\cline { 2 - 5 } & 25 & 0.8160 & 0.7576 & 0.8214 \\
\hline \multirow{5}{*}{ SCHEME_2 } & 30 & 0.7872 & 0.7282 & 0.7954 \\
\cline { 2 - 5 } & 10 & 0.8733 & 0.8486 & 0.8904 \\
\cline { 2 - 5 } & 15 & 0.8146 & 0.7847 & 0.8297 \\
\cline { 2 - 5 } & 20 & 0.7550 & 0.7252 & 0.7696 \\
\hline \multirow{3}{*}{ SCHEME_3 } & 30 & 0.6978 & 0.6685 & 0.7101 \\
\cline { 2 - 5 } & 10 & 0.6385 & 0.6147 & 0.6562 \\
\cline { 2 - 5 } & 15 & 0.8804 & 0.8023 & 0.8672 \\
\cline { 2 - 5 } & 20 & 0.8350 & 0.7237 & 0.8135 \\
\hline
\end{tabular}




\begin{tabular}{|l|l|l|l|l|}
\hline & 30 & 0.8009 & 0.6725 & 0.6942 \\
\hline \multirow{3}{*}{ SCHEME_4 } & 10 & 0.9042 & 0.8601 & 0.9169 \\
\cline { 2 - 5 } & 15 & 0.8766 & 0.8232 & 0.8865 \\
\cline { 2 - 5 } & 20 & 0.8456 & 0.7897 & 0.8578 \\
\cline { 2 - 5 } & 25 & 0.8156 & 0.7564 & 0.8293 \\
\cline { 2 - 5 } & 30 & 0.7947 & 0.7282 & 0.8045 \\
\hline
\end{tabular}

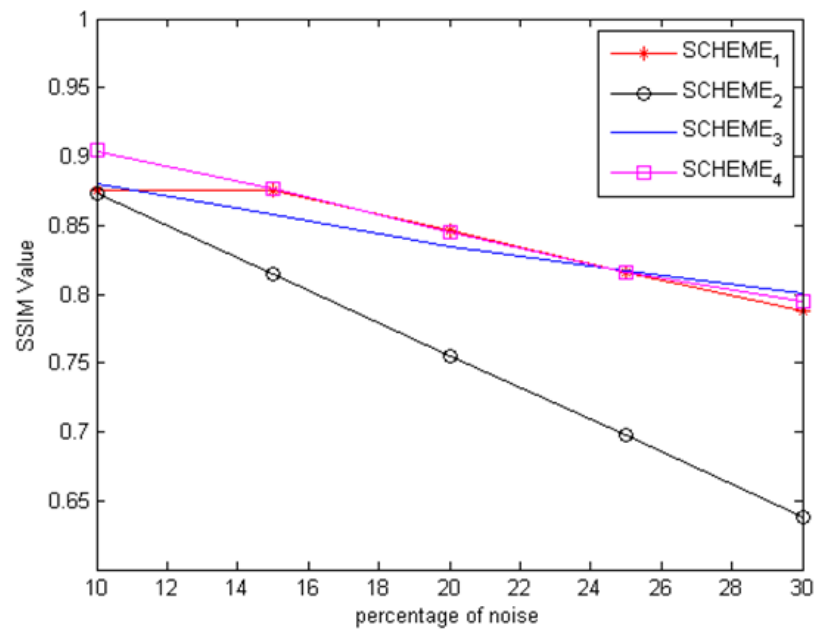

Fig. 5: MSSIM values of four denoising methods of Lena image

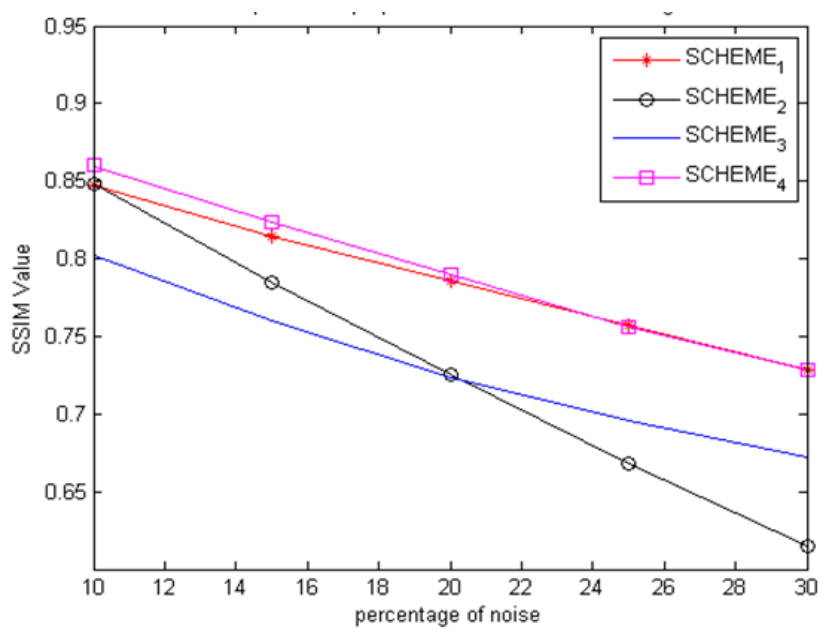

Fig. 6: MSSIM values of four denoising methods of Boat image 


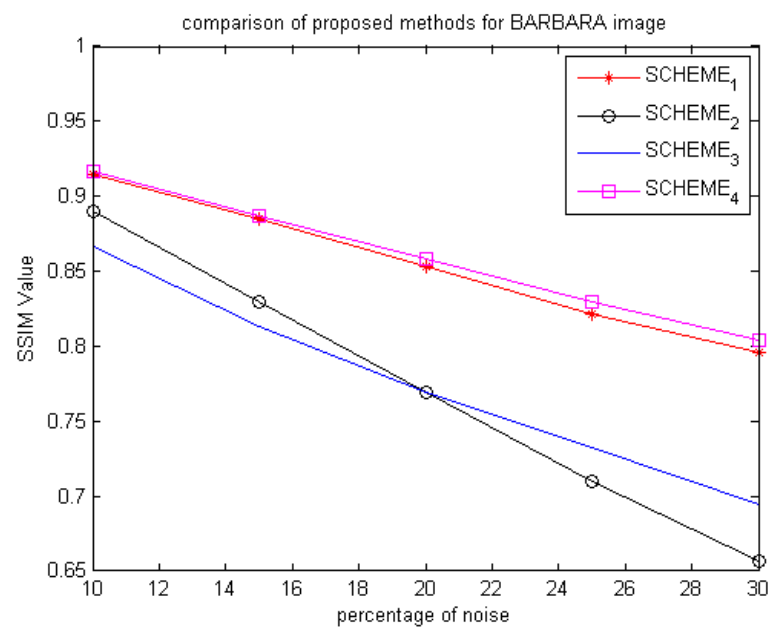

Fig. 7: MSSIM values of four denoising methods of Barbara image

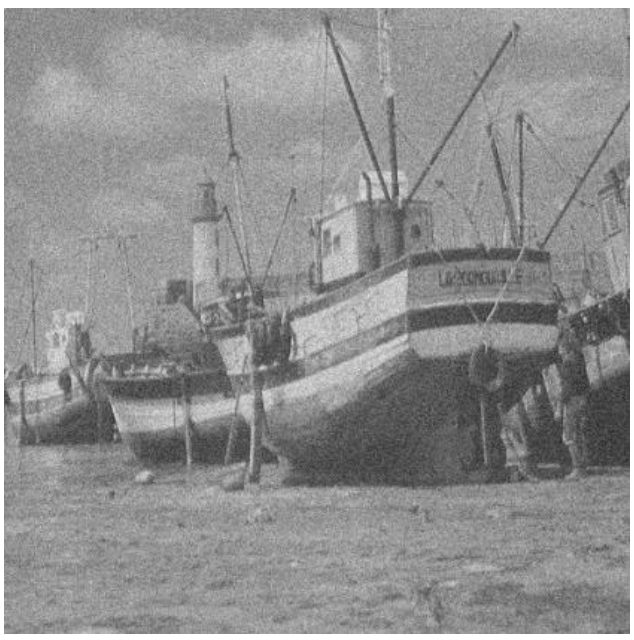

(a)

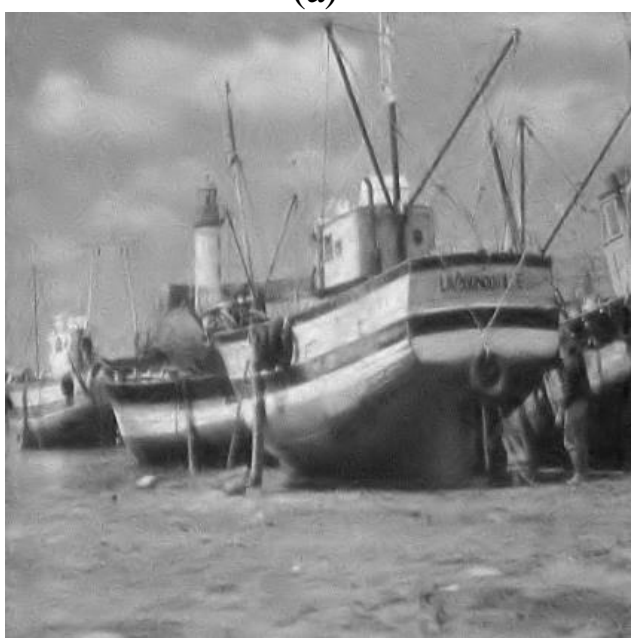

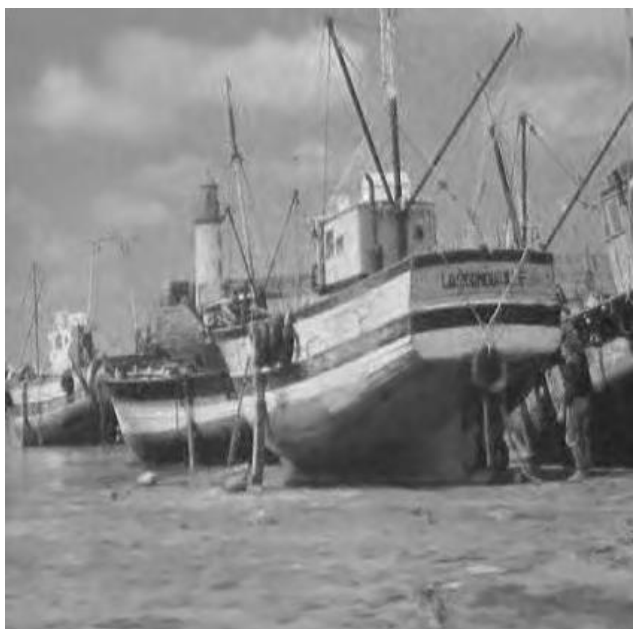

(b)

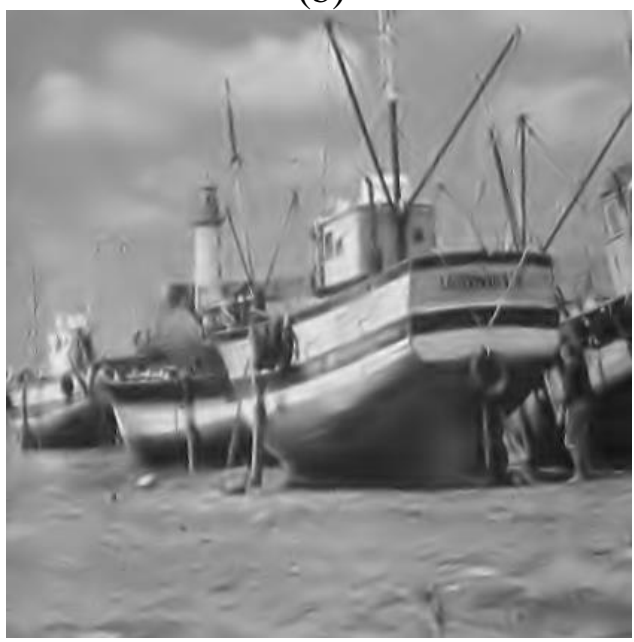


(c)

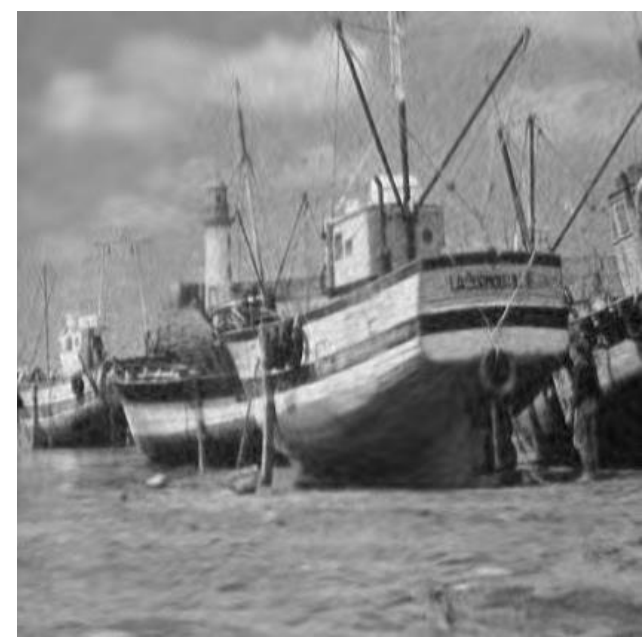

(d)

(e)

Fig. 8: Denoised image of Boat at noise power level $\sigma=25$ (a) Noisy image, (b) Scheme-1, (c) Scheme-2, (d) Scheme-3, (e) Scheme-4.

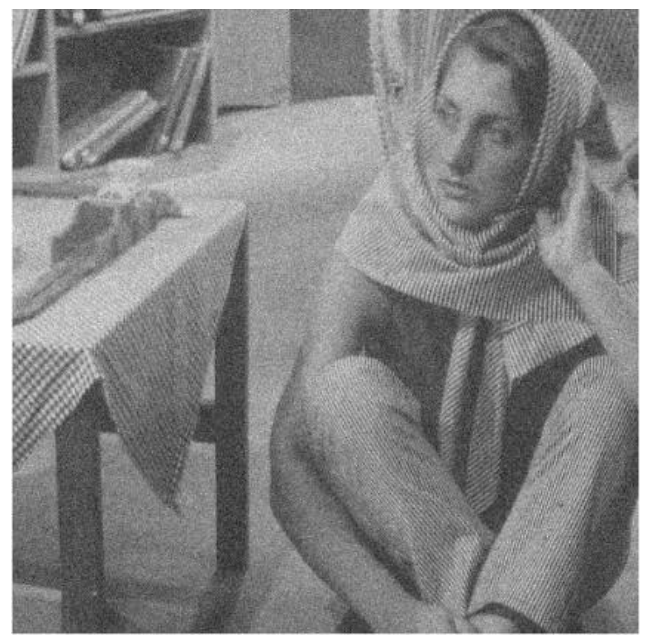

(a)

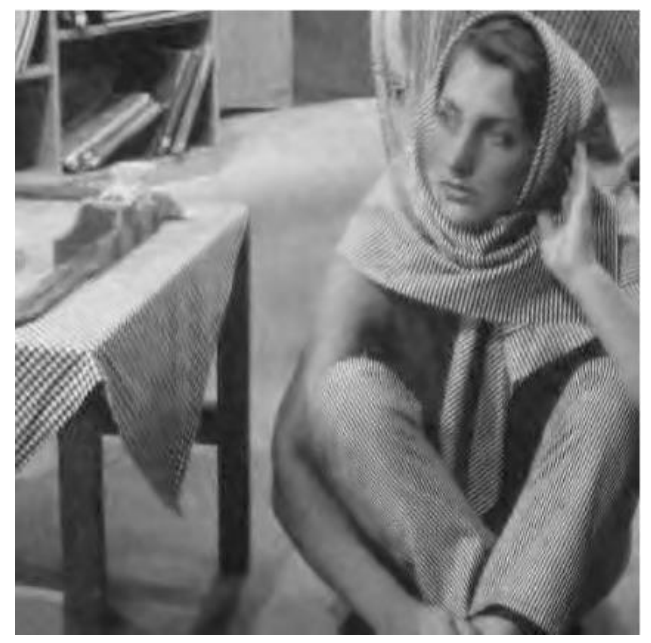

(b) 


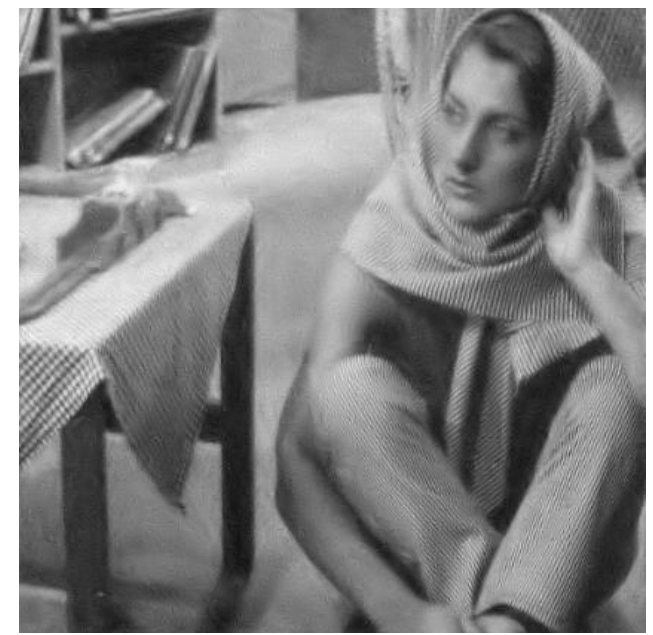

(c)

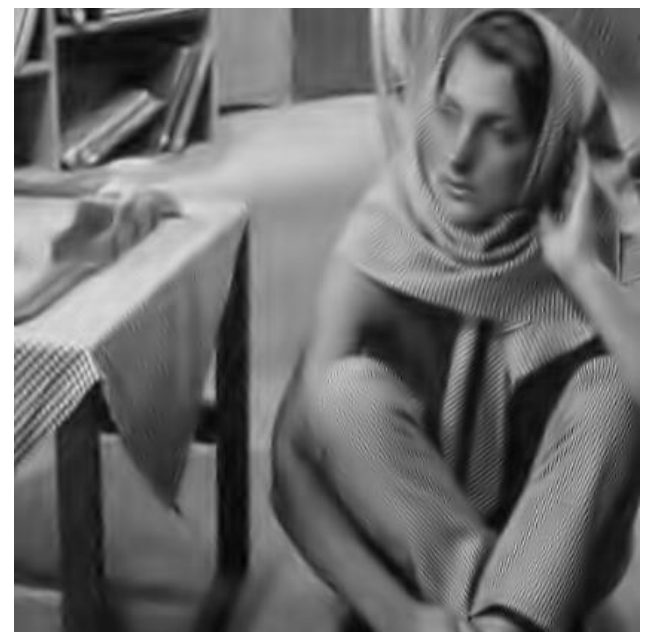

(d)

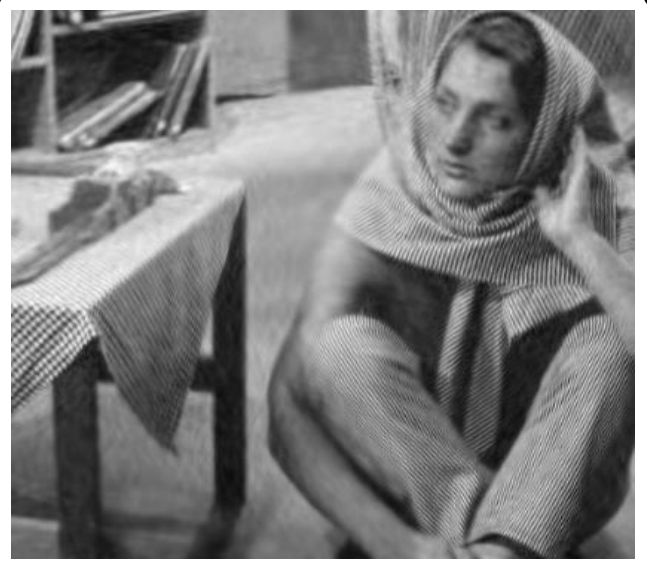

(e)

Fig. 9: Denoised image of Barbara at noise power level $\sigma=25$ (a) Noisy image, (b) Scheme-1, (c) Scheme-2, (d) Scheme-3, (e) Scheme-4.
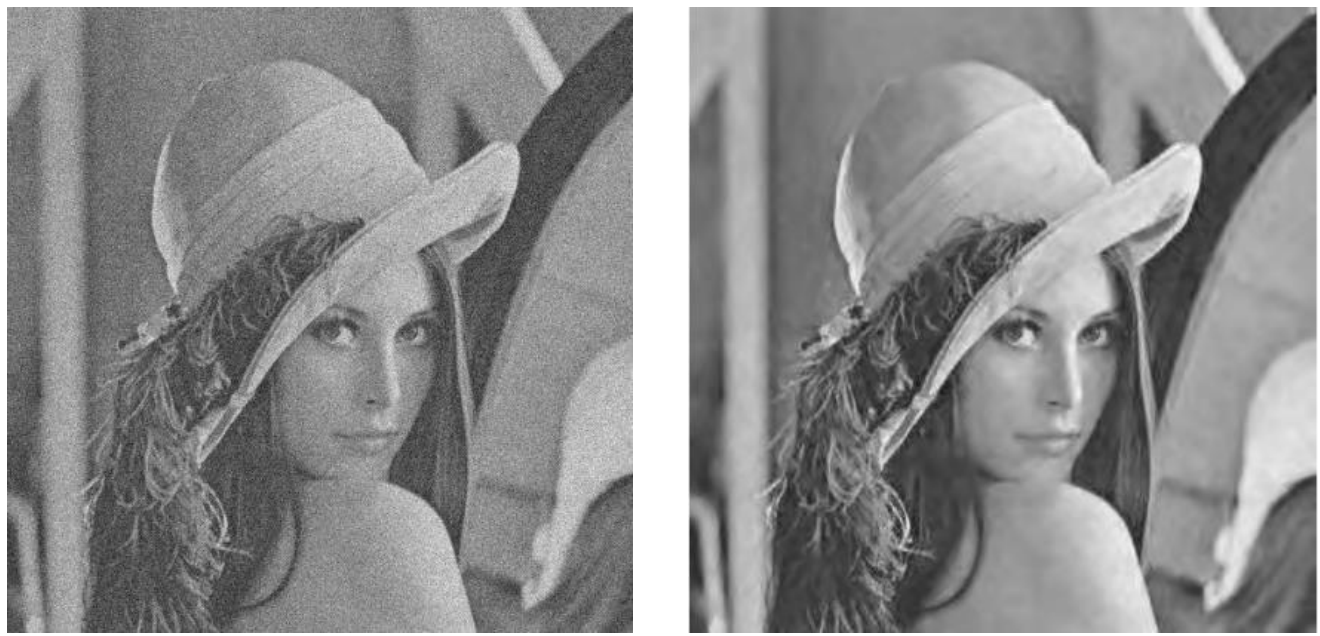

1073 
(a)

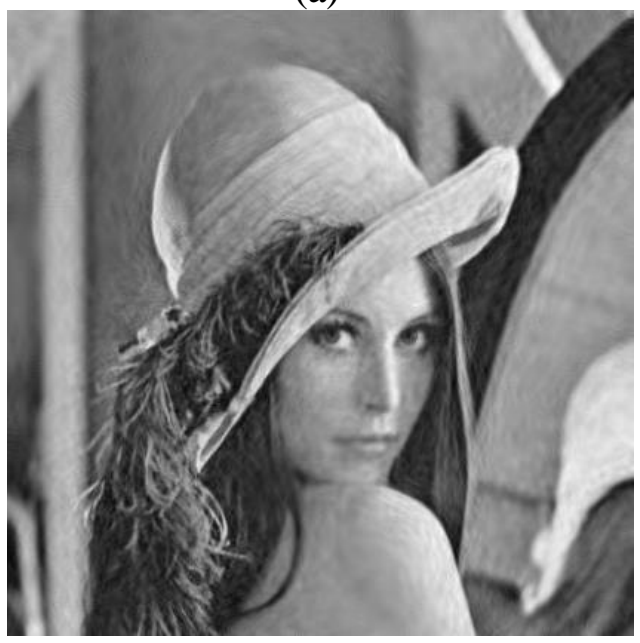

(c) (b)

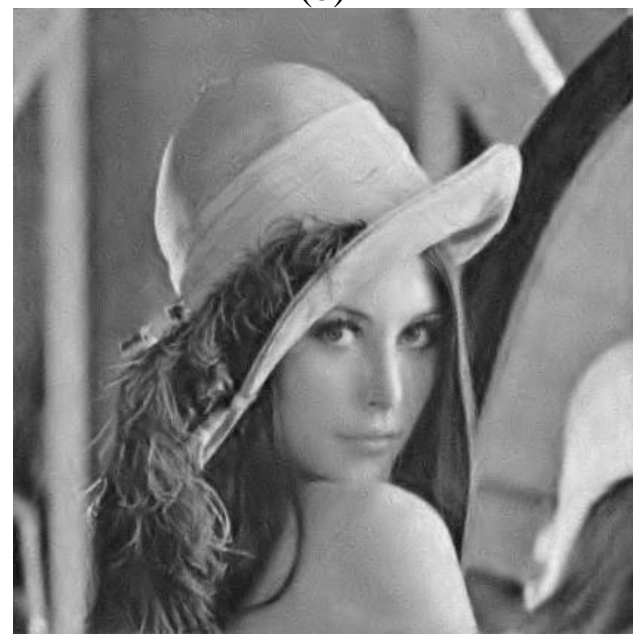

(d)

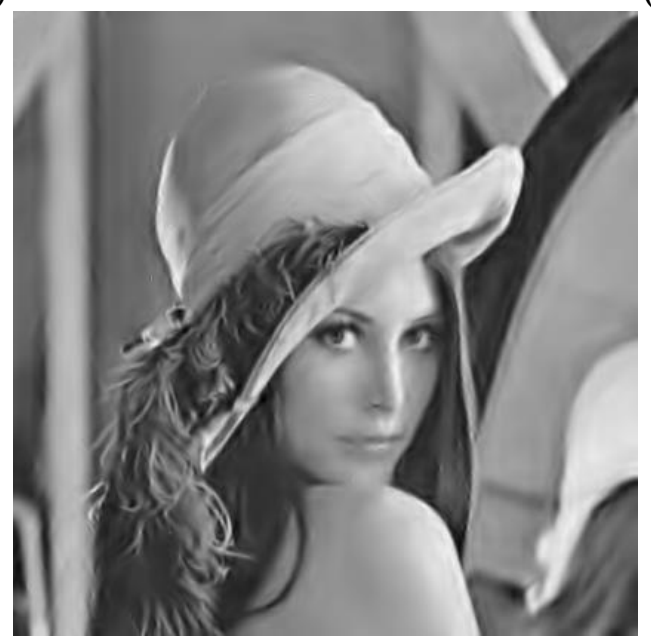

(e)

Fig. 10: Denoised image of Lena at noise power level $\sigma=25$ (a) Noisy Image, (b) Scheme-1, (c) Scheme-2, (d) Scheme-3, (e) Scheme-4.

\section{CONCLUSION}

The main aim of this paper is to study various wavelet shrinkage based image denoising techniques. Therefore this article presents a comparison between DWT, RDTDWT, DTCWT and UDTCWT based image denoising methods accompanied by the metrics PSNR, MSSIM and SSIM index map.
The first scheme is based on shrinkage scheme based minimum mean square estimator. Here MMSE depends on the noisy coefficient, its parent coefficient, noise variance and signal variance. Also NLM filter is used as a post filter that, takes the high degree of redundancy to enhance the denoising performance. The main advantage of the denoising method is sub-band adaptivity. The first scheme gives a satisfying 
result in both numerical and visual aspects.

The second scheme uses RDTDWT, it takes the advantage of redundancy present in low pass sub-band using NLM filter to suppress the low frequency noise. Also threshold value is estimated using robust median estimator and soft shrinkage is done on each coefficients, through all scale of sub-bands. The sub-band are processed separately in a loop that gives the presented scheme is a scale dependent denoising technique. The second scheme is potentially better than DWT based techniques because this scheme has approximate shift invariance.

The third scheme is based on DTCWT and VisuShrink shrinkage function is used to remove the noisy coefficient. The main modification is the noisy wavelet coefficient is computed for each scale and sub-band that gives sub-band adaptivity. The second scheme is better in noise reduction without loss of image details.

The fourth scheme uses UDTCWT that has shift invariance and directional selectivity property. In this wavelet theory wavelet coefficients and its parent are not in proper alignment. To resolve this problem, PCA is used. PCA maximizes the variability of the projected data in specific direction. Also the wavelet coefficients are shrinkage using LAWML shrinkage function. Here the shrinkage is done by processing each subband separately in a loop. The performance is measured in terms of PSNR and visual quality.

All the four denoising techniques were developed within the wavelet transform framework. The performance of denoising methods depends on sparse representation, decomposition level, threshold value, shrinkage technique, redundancy between wavelet coefficients and sub-band adaptivity. Among the four denoising techniques
UDTCWT based denoising scheme better preserve the details of the image by removing noise than other wavelet transforms.

\section{REFERENCE:}

1. Smith SM, Brady JM. SUSAN-A new approach to low level image processing. International Journal of Computer Vision 1997; 23(1):45-78. https://doi.org/10.1023/A:10079638247 $\underline{10}$

2. Tomasi C, Manduchi R. Bilateral filtering for gray and color images. Proceedings of Sixth IEEE International Conference on Computer Vision 1998. https://doi.org/10.1109/ICCV.1998.710 $\underline{815}$

3. Buades A, Coll B, Morel JM. A nonlocal algorithm for image denoising. Proceedings of IEEE International Conference on Computer Vision and Pattern Recognition 2005; 2:60-65. https://www.iro.umontreal.ca/ mignott e/IFT6150/Articles/BuadesNonLocal.pdf

4. Zhang X, Feng X, Wang W. Twodirection non-local model for image denoising. IEEE Transactions on Image Processing 2013; 22(1):408-412. https://doi.org/10.1109/TIP.2012.22140 $\underline{43}$

5. Cheng CC, Cheng FC, Huang SC, Chen $\mathrm{BH}$. Integral non-local means algorithm for image noise suppression. IET Electronics Letters 2015; 51(19):14941495 . https://doi.org/10.1049/el.2015.1829

6. Mallat SG. A theory for multiresolution signal decomposition: The wavelet representation. IEEE Transactions on Pattern Analysis and Machine Intelligence 1989; 11(7):674-693. https://doi.org/10.1109/34.192463

7. Hill PR, Achim AM, Bull DR. The undecimated dual tree complex wavelet 
transform and its application to bivariate image denoising using a Cauchy model. Proceedings of IEEE International Conference on Image Processing, 2012. https://doi.org/10.1109/ICIP.2012.6467 $\underline{082}$

8. Zhang L, Dong W, Zhang D, Shi, G. Two-stage image denoising by principal component analysis with local pixel grouping. Pattern Recognition 2010; 43:1531-1549.

https://doi.org/10.1016/j.patcog.2009.0 $\underline{9.023}$

9. Chen G, Qian S-E. Denoising of hyper spectral imagery using principal component analysis and wavelet shrinkage. IEEE Transactions on Geoscience and Remote Sensing 2011; 49(3):973-980.

https://doi.org/10.1109/TGRS.2010.20 75937

10. You SJ, Cho NI. A new image denoising method based on the wavelet domain nonlocal means filtering. Proceedings of IEEE International Conference on Acoustics, Speech and Signal Processing 2011. https://doi.org/10.1109/ICASSP.2011.5 946610

11. Balasubramanian G, Chilambuchelvan A, Vijayan S. Wavelet thresholding and joint NL Means filtering. Proceedings of IEEE International Conference on Green Computing, Communication and Conservation of Energy 2013. https://doi.org/10.1109/ICGCE.2013.68 23407 .

12. Eom IK, Kim YS. Wavelet-based denoising with nearly arbitrarily shaped windows. IEEE Signal Processing Letters 2004; 11(12):937-940. https://doi.org/10.1109/LSP.2004.8369 $\underline{40}$

13. Buades A, Coll B, Morel JM. A review of image denoising algorithms, with a new one. SIAM interdisciplinary journal: Multiscale Modeling and
Simulation $\quad 2005 ; \quad 4(2): 490-530$. https://doi.org/10.1137/040616024

14. Salmon J. On two parameters for denoising with non-local means. IEEE Signal Processing Letters 2010; 17(3):269-272.

https://doi.org/10.1109/LSP.2009.2038 $\underline{954}$

15. Xu J, Zhang L, Zuo W, Zhang D, Feng $X$. Patch group based non-local selfsimilarity prior learning for image denoising. Proceedings of IEEE International Conference on Computer Vision 2015. https://doi.org/10.1109/ICCV.2015.36

16. Tang S, Yang J. Image denoising using K-SVD and non-local means. Proceedings of IEEE Workshop on Electronics, Computer and Applications 2014.

https://doi.org/10.1109/IWECA.2014.6 845763 .

17. Yuan J. Improved anisotropic diffusion equation based on new non-local information scheme for image denoising. IET Computer Vision 2015; 9(6):864-870.

https://doi.org/10.1049/ietcvi.2014.0415

18. Elad M, Bruckstein AM. A generalized uncertainty principle and sparse representation in pairs of bases. IEEE Transactions on Information Theory 2002; 48(9):2558-2567. https://doi.org/10.1109/TIT.2002.8014 $\underline{10}$

19. Donoho DL, Johnstone IM. Adapting to unknown smoothness via wavelet shrinkage. Journal of the American Statistical Association 1995; 90(432):1200-1224. https://doi.org/10.2307/2291512

20. Donoho DL, Johnstone IM. Ideal spatial adaptation by wavelet shrinkage. Journal of Biometrika 1994; 81(3):425455. 
https://doi.org/10.1093/biomet/81.3.42 5

21. Donoho DL. Denoising by softthresholding. IEEE Transactions on Information Theory 1995; 41(3):613627. https://doi.org/10.1109/18.382009

22. Donoho DL, Johnstone IM, Kerkyacharian G, Picard D. Wavelet shrinkage: Asymptopia?. Journal of Royal Statistical Society-Series B 1995; 57(2):301-369.

https://doi.org/10.1111/j.25176161.1995.tb02032.x

23. Chang SG, Yu B, Vetterli M, Adaptive wavelet thresholding for image denoising and compression. IEEE Transactions on Image Processing 2000; 9(9):1532-1546. https://doi.org/10.1109/83.862633

24. Kingsbury NG. The dual-tree complex wavelet transform: A new technique for shift invariance and directional filters. Proceedings of 8th IEEE DSP Workshop 1998; 86:9-12. https://pdfs.semanticscholar.org/294e/f 14804eeb9bed629eab9ff974ca3d0ffe92 a.pdf

25. Mihcak MK, Kozintsev I, Ramchandran $\mathrm{K}$, Moulin P. Low-complexity image denoising based on statistical modeling of wavelet coefficients. IEEE Signal Processing Letters 1999; 6(12):300303. https://doi.org/10.1109/97.803428

26. Sendur L, Selesnick IW. Bivariate shrinkage functions for wavelet-based denoising exploiting interscale dependency. IEEE Transactions on Signal Processing 2002; 50(11):27442756.

https://doi.org/10.1109/TSP.2002.8040 $\underline{91}$

27. Sendur L, Selesnick IW. Bivariate shrinkage with local variance estimation. IEEE Signal Processing Letters 2002; 9(12):438-441. https://doi.org/10.1109/LSP.2002.8060 $\underline{54}$
28. Rabbani H, Gazor S. Image denoising employing local mixture models in sparse domains. IET Image Processing 2010; 4(5):413-428. https://doi.org/10.1049/ietipr.2009.0048

29. Hussain SA, Gorashi SM. Image denoising algorithm based on hybrid neighborhood filter. Proceedings of IEEE International Conference on Signal and Image Processing Applications 2013. https://doi.org/10.1109/ICSIPA.2013.6 $\underline{707972}$

30. Unser M. Texture classification and segmentation using wavelet frames. IEEE Transaction on Image Processing 1995; 4:1549-1560. https://doi.org/10.1109/83.469936

31. Rockinger O. Pixel-level fusion of image sequences using wavelet frames. Proceedings of Leeds Applied Shape Research Workshop 1996. https://pdfs.semanticscholar.org/6e11/5 cdb2558120ff326138cd92bb19768ce06 de.pdf

32. Gyaourova A. Undecimated wavelet transforms for image denoising. Center for Applied Scientific Computing, Lawrence Livermore National Laboratory, Canada, 2012. https://pdfs.semanticscholar.org/0a8c/3 61d27bd26ce874f79889da39cef5d6a05 $\underline{51 . p d f}$

33. Wang W, Li Y. Bayesian denoising for remote sensing image based on undecimated discrete wavelet transform. Proceedings of IEEE International Conference on Information Engineering and Computer Science 2009. https://doi.org/10.1109/ICIECS.2009.5 $\underline{365574}$

34. Achim AM, Kuruoglu EE. Image denoising using bivariate $\alpha$-stable distributions in the complex wavelet domain. IEEE Signal Processing Letters 
2005;

12:17-20

https://doi.org/10.1109/LSP.2004.8396

$\underline{92}$

35. Liu $Y$, Jiang $M$. Image denoising algorithm based on DTCWT and adaptive windows. Proceedings of IEEE International Conference on Computer Science and Information Technology 2010.

https://doi.org/10.1109/ICCSIT.2010.5 564033

36. Chen G, Zhu W-P, Xie W. Waveletbased image denoising using three scales of dependency. IET Image Processing 2012; 6(6):756-760. https://doi.org/10.1049/ietipr.2010.0408

37. Varsha A, Preetha B. An improved dual tree complex wavelet transform based image denoising using GCV thresholding. Proceedings of IEEE International Conference on Computational Systems and Communications 2014. https://doi.org/10.1109/COMPSC.2014 .7032635

38. Zhang $X$. Image denoising using dualtree complex wavelet transform and Wiener filter with modified thresholding. Journal of Scientific \& Industrial Research 2016; 75(11):687690.

http://nopr.niscair.res.in/handle/123456 789/36888

39. Yaseen AS, Pavlova ON Pavlov AN, Hramov AE. Image denoising with the dual-tree complex wavelet transform. Proceedings of SPIE 2016; 9917. https://doi.org/10.1117/12.2225449

40. Ramadhan A, Mahmood F, Elci A. Image denoising by median filter in wavelet domain. The International Journal of Multimedia \& Its Applications 2017; 9(1):31-40. https://doi.org/10.5121/ijma.2017.9104

41. Gajbhar SS, Joshi MV. Image denoising using tight-frame dual-tree complex wavelet transform. Machine Intelligence and Signal Analysis, Advances in Intelligent Systems and Computing 2018; 748:645-656. https://doi.org/10.1007/978-981-130923-6_55

42. Vijayaraghavan V, Laavanya $M$, Karthikeyan M. Real oriented 2-D dual tree wavelet transform with non-local means filter for image denoising. Journal of Electrical Engineering 2017; 17(2):106-111.

http://jee.ro/covers/art.php?issue=WC1 457629879W56e1aab781769

43. Vijayaraghavan V, Karthikeyan M. Amalgamation of dual tree complex wavelet transform and non-local means filter with visushrink for image denoising. International Journal of Printing, Packaging \& Allied Sciences 2016; 4(3):1890-1894.

44. Vijayaraghavan V, Karthikeyan M. Wavelet based image denoising with locally adaptive window and non-local means filter. Asian Journal of Research in Social Sciences and Humanities 2017; 7(3):1080-1086. https://doi.org/10.5958/22497315.2017.00229.5

45. Vijayaraghavan V, Karthikeyan M. Denoising of images using principal component analysis and undecimated dual tree complex wavelet transform. International Journal of Biomedical Engineering and Technology, Inderscience 2018; 26(3-4):304-

315. https://doi.org/10.1504/IJBET.201 $\underline{8.089962}$ 